\title{
Improvement of the Modified Decomposition Method for Handling Third-Order Singular Nonlinear Partial Differential Equations with Applications in Physics
}

\author{
Nemat Dalir \\ Department of Mechanical Engineering, Salmas Branch, Islamic Azad University, Salmas, Iran \\ Correspondence should be addressed to Nemat Dalir; dalir@aut.ac.ir
}

Received 4 June 2014; Accepted 9 October 2014; Published 6 November 2014

Academic Editor: Athanasios N. Yannacopoulos

Copyright (c) 2014 Nemat Dalir. This is an open access article distributed under the Creative Commons Attribution License, which permits unrestricted use, distribution, and reproduction in any medium, provided the original work is properly cited.

\begin{abstract}
The modified decomposition method (MDM) is improved by introducing new inverse differential operators to adapt the MDM for handling third-order singular nonlinear partial differential equations (PDEs) arising in physics and mechanics. A few casestudy singular nonlinear initial-value problems (IVPs) of third-order PDEs are presented and solved by the improved modified decomposition method (IMDM). The solutions are compared with the existing exact analytical solutions. The comparisons show that the IMDM is effectively capable of obtaining the exact solutions of the third-order singular nonlinear IVPs.
\end{abstract}

\section{Introduction}

Singular nonlinear PDEs appear in many cases in physics and mechanics. Examples of singular nonlinear PDEs in physics include cylindrical and spherical KdV equations, Ernst equation, Clairaut's equation, Hartree equation, Yamabe problem, Zakharov-Schulman system, Cauchy momentum equation, and reaction-diffusion equations [1-3]. Examples of singular nonlinear PDEs in mechanics are equation of motion of a point mass in a central force field, generalized equation of steady transonic gas flow, cylindrical and spherical NavierStokes equations, and cylindrical and spherical fluid hydrodynamic instability equations $[4,5]$. However, despite such importance in various fields of science and engineering, singular nonlinear PDEs are difficult to solve.

In recent years, semianalytical methods such as the Adomian decomposition method (ADM) and the modified decomposition method (MDM) have gained significance in solving many problems in physics and mathematics. ODEs and PDEs of various types have been solved by the MDM, such that singular and nonsingular nonlinear ODEs and also nonsingular nonlinear PDEs have been solved by the MDM [6-10]. Nevertheless, literature survey makes it clear that the MDM is never attempted on solving the singular nonlinear PDEs. Wazwaz [11] and Wazwaz and Mehanna
[12] used the combined Laplace transform-ADM for handling nonlinear Volterra integrodifferential equations and singular integral equation of heat transfer, respectively. Sivakumar and Baiju [13] used a shooting type Laplace-Adomian decomposition algorithm for solving nonlinear differential equations with boundary conditions at infinity. Noghrehabadi et al. [14] combined Pade approximants and the ADM to study the deflection and pull-in instability of nanocantilever electromechanical switches. Duan and Rach [15] developed new numerical modified Adomian decomposition algorithms by using the Wazwaz-El-Sayed modified decomposition recursion scheme and investigated their practicality and efficiency for several nonlinear examples. Lin et al. [16], based on the new definition of the Adomian polynomials, the ADM, and the Pade approximants technique, proposed a new algorithm to construct analytical approximate solutions for nonlinear fractional differential equations with initial or boundary conditions. Kermani and Dehestani [17] applied the ADM to solve the nonlinear equations from the 1D model for a nanosized oscillator. Song and Wang [18] introduced a convergence-control parameter into standard ADM and established a new iterative formula.

In the present study, the goal is to develop new inverse differential operators incorporated into the MDM to solve the third-order singular nonlinear PDEs. Thus the improved 
modified decomposition method (IMDM), which is the MDM in conjunction with the new developed inverse differential operators, is used to solve the singular nonlinear IVPs in the third-order PDEs. The results of the IMDM solutions agree with the existing exact solutions of IVPs.

\section{IMDM for Third-Order Singular Nonlinear PDEs}

2.1. Third-Order Singular Nonlinear PDEs. We consider the third-order, in $t$, singular nonlinear PDE as follows:

$$
\frac{\partial^{3} u}{\partial t^{3}}+\frac{n}{t} \frac{\partial^{2} u}{\partial t^{2}}=F\left(x, u, \frac{\partial u}{\partial x}, \frac{\partial^{2} u}{\partial x^{2}}, \frac{\partial u}{\partial t}\right)
$$

where $t$ and $x$ are independent variables, $u$ is the dependent variable, $F$ is a nonlinear function of $x, u, u_{x}, u_{x x}$, and $u_{t}$, and $n$ is a real constant, $n>0$. The initial conditions are as follows:

$$
\begin{gathered}
u(x, 0)=f(x), \quad u_{t}(x, 0)=g(x) \\
u_{t t}(x, 0)=h(x)
\end{gathered}
$$

By defining the linear differential operator $L_{t}(\cdot)=\partial^{3}(\cdot) / \partial t^{3}+$ $(n / t)\left(\partial^{2}(\cdot) / \partial t^{2}\right)$, the left hand side of (1) becomes as $L_{t} u=$ $\partial^{3} u / \partial t^{3}+(n / t)\left(\partial^{2} u / \partial t^{2}\right)$. Then, (1) can be rewritten as

$$
L_{t} u=F\left(x, u, \frac{\partial u}{\partial x}, \frac{\partial^{2} u}{\partial x^{2}}, \frac{\partial u}{\partial t}\right)
$$

The inverse of operator $L_{t}$, that is, $L_{t}^{-1}$, is defined such that $L_{t}^{-1}\left\{L_{t}(u)\right\}=u(x, t)-u(x, 0)-t u_{t}(x, 0)$ in the following form:

$$
L_{t}^{-1}(\cdot)=\iint_{0}^{t} \frac{1}{t^{n}} \int_{0}^{t} t^{n}(\cdot) d t d t d t
$$

It can be shown in the following manner that the application of $L_{t}^{-1}$ on $\partial^{3} u / \partial t^{3}+(n / t)\left(\partial^{2} u / \partial t^{2}\right)$ gives $u(x, t)-u(x, 0)-$ $t u_{t}(x, 0)$ :

$$
\begin{aligned}
& L_{t}^{-1}\left(\frac{\partial^{3} u}{\partial t^{3}}+\frac{n}{t} \frac{\partial^{2} u}{\partial t^{2}}\right) \\
& \quad=\iint_{0}^{t} \frac{1}{t^{n}} \int_{0}^{t} t^{n}\left(\frac{\partial^{3} u}{\partial t^{3}}+\frac{n}{t} \frac{\partial^{2} u}{\partial t^{2}}\right) d t d t d t \\
& =\iint_{0}^{t} \frac{1}{t^{n}} \int_{0}^{t}\left(t^{n} \frac{\partial^{3} u}{\partial t^{3}}+n t^{n-1} \frac{\partial^{2} u}{\partial t^{2}}\right) d t d t d t \\
& =\iint_{0}^{t} \frac{1}{t^{n}} \int_{0}^{t} \frac{\partial}{\partial t}\left(t^{n} \frac{\partial^{2} u}{\partial t^{2}}\right) d t d t d t \\
& =\iint_{0}^{t} \frac{1}{t^{n}}\left(t^{n} \frac{\partial^{2} u}{\partial t^{2}}\right)_{0}^{t} d t d t
\end{aligned}
$$

$$
\begin{aligned}
& =\iint_{0}^{t} \frac{1}{t^{n}}\left(t^{n} \frac{\partial^{2} u}{\partial t^{2}}\right) d t d t \\
& =\iint_{0}^{t} \frac{\partial}{\partial t}\left(\frac{\partial u}{\partial t}\right) d t d t=\int_{0}^{t}\left(\frac{\partial u}{\partial t}\right)_{0}^{t} d t \\
& =\int_{0}^{t}\left(\frac{\partial u}{\partial t}-\left.\frac{\partial u}{\partial t}\right|_{(x, 0)}\right) d t \\
& =\int_{0}^{t}\left(\frac{\partial u}{\partial t}\right) d t-\int_{0}^{t}\left(\left.\frac{\partial u}{\partial t}\right|_{(x, 0)}\right) d t \\
& =(u)_{0}^{t}-\left.t \frac{\partial u}{\partial t}\right|_{(x, 0)}=u(x, t)-u(x, 0)-t u_{t}(x, 0) .
\end{aligned}
$$

The inverse differential operator of (4), proposed in the present work, introduces the improved modified decomposition method (IMDM) for solving third-order singular nonlinear PDEs. Applying the inverse differential operator of (4) to (3) and use of the boundary conditions of (2) gives:

$$
u(x, t)=f(x)+\operatorname{tg}(x)+L_{t}^{-1}\left(F\left(x, u, \frac{\partial u}{\partial x}, \frac{\partial^{2} u}{\partial x^{2}}, \frac{\partial u}{\partial t}\right)\right) .
$$

The ADM and MDM state that the dependent variable $u(x, t)$ and the nonlinear terms $F$ in (6) should be substituted with the following infinite series [7]:

$$
\begin{gathered}
u(x, t)=\sum_{m=0}^{\infty} u_{m}(x, t), \\
F\left(x, u, \frac{\partial u}{\partial x}, \frac{\partial^{2} u}{\partial x^{2}}, \frac{\partial u}{\partial t}\right)=\sum_{m=0}^{\infty} A_{m}(x, t),
\end{gathered}
$$

where $A_{m}$ 's, called the Adomian polynomials, are defined as [8]

$$
A_{m}=\left[\frac{1}{m !} \frac{d^{m}}{d \lambda^{m}} F\left(\sum_{i=0}^{m} \lambda^{i} u_{i}\right)\right]_{\lambda=0} .
$$

Substitution of infinite series of (7) in (6) results in

$$
\sum_{m=0}^{\infty} u_{m}(x, t)=f(x)+\operatorname{tg}(x)+L_{t}^{-1}\left(\sum_{m=0}^{\infty} A_{m}(x, t)\right) .
$$

Due to the ADM, all terms of $u(x, t)$ except $u_{0}(x, t)$ are determined by a recursive relation, as follows [9]:

$$
\begin{gathered}
u_{0}(x, t)=f(x)+\operatorname{tg}(x), \\
u_{1}(x, t)=L_{t}^{-1}\left(A_{0}(x, t)\right), \\
u_{m+1}(x, t)=L_{t}^{-1}\left(A_{m}(x, t)\right), \quad m \geq 1 .
\end{gathered}
$$

In MDM, a slight modification is applied to the ADM to enhance the convergence behavior of decomposition method, such that $f(x)+\operatorname{tg}(x)$ is split into two parts; the first part, 
$f(x)$, is written with $u_{0}(x, t)$, and the second part, $\operatorname{tg}(x)$, is written with $u_{1}(x, t)$ as follows [10]:

$$
\begin{gathered}
u_{0}(x, t)=f(x), \\
u_{1}(x, t)=\operatorname{tg}(x)+L_{t}^{-1}\left(A_{0}(x, t)\right), \\
u_{m+1}(x, t)=L_{t}^{-1}\left(A_{m}(x, t)\right), \quad m \geq 1 .
\end{gathered}
$$

2.2. General Third-Order Singular Nonlinear PDEs. We take into account the general third-order (in $t$ ) singular nonlinear PDE as follows:

$$
\frac{\partial^{3} u}{\partial t^{3}}+\frac{2 n}{t} \frac{\partial^{2} u}{\partial t^{2}}+\frac{n(n-1)}{t^{2}} \frac{\partial u}{\partial t}=F\left(x, u, \frac{\partial u}{\partial x}, \frac{\partial^{2} u}{\partial x^{2}}, \frac{\partial u}{\partial t}\right)
$$

where $t$ and $x$ are independent variables, $u$ is the dependent variable, $F$ is a nonlinear function of $x, u, u_{x}, u_{x x}$, and $u_{t}$, and $n$ is a real constant, $n>1$. The initial conditions are as follows:

$$
\begin{gathered}
u(x, 0)=f(x), \quad u_{t}(x, 0)=g(x), \\
u_{t t}(x, 0)=h(x)
\end{gathered}
$$

By defining the linear differential operator $L_{t}(\cdot)=\partial^{3}(\cdot) / \partial t^{3}+$ $(2 n / t)\left(\partial^{2}(\cdot) / \partial t^{2}\right)+\left(n(n-1) / t^{2}\right)(\partial(\cdot) / \partial t)$, the left hand side of (12) is rewritten as $L_{t} u=\partial^{3} u / \partial t^{3}+(2 n / t)\left(\partial^{2} u / \partial t^{2}\right)+(n(n-$ $\left.1) / t^{2}\right)(\partial u / \partial t)$. Thus, (12) becomes as

$$
L_{t} u=F\left(x, u, \frac{\partial u}{\partial x}, \frac{\partial^{2} u}{\partial x^{2}}, \frac{\partial u}{\partial t}\right) .
$$

The $L_{t}^{-1}$ is defined in the following form such that $L_{t}^{-1}\left\{L_{t}(u)\right\}=u(x, t)-u(x, 0)$ :

$$
L_{t}^{-1}(\cdot)=\int_{0}^{t} \frac{1}{t^{n}} \iint_{0}^{t} t^{n}(\cdot) d t d t d t .
$$

It can be shown in the following manner that $L_{t}^{-1}\left\{L_{t}(u)\right\}=$ $u(x, t)-u(x, 0)$

$$
\begin{aligned}
& L_{t}^{-1}\left(\frac{\partial^{3} u}{\partial t^{3}}+\frac{2 n}{t} \frac{\partial^{2} u}{\partial t^{2}}+\frac{n(n-1)}{t^{2}} \frac{\partial u}{\partial t}\right) \\
& =\int_{0}^{t} \frac{1}{t^{n}} \iint_{0}^{t} t^{n}\left(\frac{\partial^{3} u}{\partial t^{3}}+\frac{2 n}{t} \frac{\partial^{2} u}{\partial t^{2}}+\frac{n(n-1)}{t^{2}} \frac{\partial u}{\partial t}\right) d t d t d t \\
& =\int_{0}^{t} \frac{1}{t^{n}} \iint_{0}^{t}\left(t^{n} \frac{\partial^{3} u}{\partial t^{3}}+2 n t^{n-1} \frac{\partial^{2} u}{\partial t^{2}}\right. \\
& =\int_{0}^{t} \frac{1}{t^{n}} \iint_{0}^{t} \frac{\partial}{\partial t}\left(t^{n} \frac{\partial^{2} u}{\partial t^{2}}+n t^{n-1} \frac{\partial u}{\partial t}\right) d t d t d t \\
& =\int_{0}^{t} \frac{1}{t^{n}} \int_{0}^{t}\left(t^{n} \frac{\partial^{2} u}{\partial t^{2}}+n t^{n-1} \frac{\partial u}{\partial t}\right)_{0}^{t} d t d t
\end{aligned}
$$

$$
\begin{aligned}
& =\int_{0}^{t} \frac{1}{t^{n}} \int_{0}^{t}\left(t^{n} \frac{\partial^{2} u}{\partial t^{2}}+n t^{n-1} \frac{\partial u}{\partial t}\right) d t d t \\
& =\int_{0}^{t} \frac{1}{t^{n}} \int_{0}^{t} \frac{\partial}{\partial t}\left(t^{n} \frac{\partial u}{\partial t}\right) d t d t \\
& =\int_{0}^{t} \frac{1}{t^{n}}\left(t^{n} \frac{\partial u}{\partial t}\right)_{0}^{t} d t=\int_{0}^{t} \frac{1}{t^{n}}\left(t^{n} \frac{\partial u}{\partial t}\right) d t \\
& =\int_{0}^{t}\left(\frac{\partial u}{\partial t}\right) d t=(u)_{0}^{t}=u(x, t)-u(x, 0) .
\end{aligned}
$$

The inverse differential operator of (15), defined in the present work, can be used in conjunction with the MDM to solve the general third-order singular nonlinear PDEs. Applying the inverse operator of (15) to the differential equation (14) results in

$$
u(x, t)=f(x)+L_{t}^{-1}\left(F\left(x, u, \frac{\partial u}{\partial x}, \frac{\partial^{2} u}{\partial x^{2}}, \frac{\partial u}{\partial t}\right)\right) .
$$

Substituting the infinite series of (7) in (17), due to the MDM, gives the following form [9]:

$$
\sum_{m=0}^{\infty} u_{m}(x, t)=f(x)+L_{t}^{-1}\left(\sum_{m=0}^{\infty} A_{m}(x, t)\right),
$$

where the Adomian polynomials $A_{m}$ 's are defined in (8). The MDM form of (18) can be written as [10]

$$
\begin{gathered}
u_{0}(x, t)=f(x), \\
u_{1}(x, t)=L_{t}^{-1}\left(A_{0}(x, t)\right), \\
u_{m+1}(x, t)=L_{t}^{-1}\left(A_{m}(x, t)\right), \quad m \geq 1 .
\end{gathered}
$$

2.3. General Complete Third-Order Singular Nonlinear PDEs. We consider the general complete third-order, in $t$, singular nonlinear PDE as follows:

$$
\begin{aligned}
& \frac{\partial^{3} u}{\partial t^{3}}+\frac{3 n}{t} \frac{\partial^{2} u}{\partial t^{2}}+\frac{3 n(n-1)}{t^{2}} \frac{\partial u}{\partial t}+\frac{n(n-1)(n-2)}{t^{3}} u \\
& =F\left(x, u, \frac{\partial u}{\partial x}, \frac{\partial^{2} u}{\partial x^{2}}, \frac{\partial u}{\partial t}\right),
\end{aligned}
$$

where $t$ and $x$ are independent variables, $u$ is the dependent variable, $F$ is a nonlinear function of $x, u, u_{x}, u_{x x}$, and $u_{t}$, and $n$ is a real constant, $n>2$. The initial conditions are as

$$
\begin{gathered}
u(x, 0)=f(x), \quad u_{t}(x, 0)=g(x), \\
u_{t t}(x, 0)=h(x) .
\end{gathered}
$$

By defining the linear operator $L_{t}(\cdot)=\partial^{3}(\cdot) / \partial t^{3}+$ $(3 n / t)\left(\partial^{2}(\cdot) / \partial t^{2}\right)+\left(3 n(n-1) / t^{2}\right)(\partial(\cdot) / \partial t)+(n(n-1)(n-$ 2) $\left./ t^{3}\right)(\cdot)$, the left hand side of $(20)$ is rewritten as $L_{t} u=$ $\partial^{3} u / \partial t^{3}+(3 n / t)\left(\partial^{2} u / \partial t^{2}\right)+\left(3 n(n-1) / t^{2}\right)(\partial u / \partial t)+(n(n-$ 1) $\left.(n-2) / t^{3}\right) u$, such that $(20)$ becomes as

$$
L_{t} u=F\left(x, u, \frac{\partial u}{\partial x}, \frac{\partial^{2} u}{\partial x^{2}}, \frac{\partial u}{\partial t}\right) .
$$


The inverse operator of $L_{t}$, that is, $L_{t}^{-1}$, is defined in the following form such that $L_{t}^{-1}\left\{L_{t}(u)\right\}=u$ :

$$
L_{t}^{-1}(\cdot)=\frac{1}{t^{n}} \iiint_{0}^{t} t^{n}(\cdot) d t d t d t
$$

The credibility of definition of the inverse operator $L_{t}^{-1},(23)$, is shown in the following manner:

$$
\begin{aligned}
& L_{t}^{-1}\left(\frac{\partial^{3} u}{\partial t^{3}}+\frac{3 n}{t} \frac{\partial^{2} u}{\partial t^{2}}+\frac{3 n(n-1)}{t^{2}} \frac{\partial u}{\partial t}\right. \\
& \left.+\frac{n(n-1)(n-2)}{t^{3}} u\right) \\
& =\frac{1}{t^{n}} \iiint_{0}^{t} t^{n}\left(\frac{\partial^{3} u}{\partial t^{3}}+\frac{3 n}{t} \frac{\partial^{2} u}{\partial t^{2}}+\frac{3 n(n-1)}{t^{2}} \frac{\partial u}{\partial t}\right. \\
& \left.+\frac{n(n-1)(n-2)}{t^{3}} u\right) d t d t d t \\
& =\frac{1}{t^{n}} \iiint_{0}^{t}\left(t^{n} \frac{\partial^{3} u}{\partial t^{3}}+3 n t^{n-1} \frac{\partial^{2} u}{\partial t^{2}}\right. \\
& +3 n(n-1) t^{n-2} \frac{\partial u}{\partial t} \\
& \left.+n(n-1)(n-2) t^{n-3} u\right) d t d t d t \\
& =\frac{1}{t^{n}} \iiint_{0}^{t} \frac{\partial}{\partial t}\left(t^{n} \frac{\partial^{2} u}{\partial t^{2}}+2 n t^{n-1} \frac{\partial u}{\partial t}\right. \\
& \left.+n(n-1) t^{n-2} u\right) d t d t d t \\
& =\frac{1}{t^{n}} \iint_{0}^{t}\left(t^{n} \frac{\partial^{2} u}{\partial t^{2}}+2 n t^{n-1} \frac{\partial u}{\partial t}\right. \\
& \left.+n(n-1) t^{n-2} u\right) d t d t \\
& =\frac{1}{t^{n}} \iint_{0}^{t} \frac{\partial}{\partial t}\left(t^{n} \frac{\partial u}{\partial t}+n t^{n-1} u\right) d t d t \\
& =\frac{1}{t^{n}} \int_{0}^{t}\left(t^{n} \frac{\partial u}{\partial t}+n t^{n-1} u\right)_{0}^{t} d t \\
& =\frac{1}{t^{n}} \int_{0}^{t}\left(t^{n} \frac{\partial u}{\partial t}+n t^{n-1} u\right) d t \\
& =\frac{1}{t^{n}} \int_{0}^{t} \frac{\partial}{\partial t}\left(t^{n} u\right) d t=\frac{1}{t^{n}}\left(t^{n} u\right)_{0}^{t} \\
& =\frac{1}{t^{n}}\left(t^{n} u\right)=u(x, t) \text {. }
\end{aligned}
$$

The inverse operator of (23), which is developed in the present work, improves the MDM for solving the third-order singular nonlinear PDEs. Application of the inverse operator of (23) on (22) results in

$$
u(x, t)=L_{t}^{-1}\left(F\left(x, u, \frac{\partial u}{\partial x}, \frac{\partial^{2} u}{\partial x^{2}}, \frac{\partial u}{\partial t}\right)\right) .
$$

Substitution of the infinite series of (7) in (25) gives the following form [9]:

$$
\sum_{m=0}^{\infty} u_{m}(x, t)=L_{t}^{-1}\left(\sum_{m=0}^{\infty} A_{m}(x, t)\right)
$$

where $A_{m}$ 's are defined in (8). The MDM for (26) can be written as [10]

$$
\begin{gathered}
u_{0}(x, t)=0, \\
u_{1}(x, t)=L_{t}^{-1}\left(A_{0}(x, t)\right), \\
u_{m+1}(x, t)=L_{t}^{-1}\left(A_{m}(x, t)\right), \quad m \geq 1 .
\end{gathered}
$$

\section{Case Studies of Third-Order Singular Nonlinear PDEs Solved by IMDM}

3.1. Case Study 1. We consider the following singular nonlinear initial-value problem (IVP) of PDE:

$$
\begin{gathered}
\frac{\partial^{3} u}{\partial t^{3}}+\frac{4}{t} \frac{\partial^{2} u}{\partial t^{2}}+\frac{2}{t^{2}} \frac{\partial u}{\partial t}=\frac{\partial^{3} u}{\partial t^{3}} \cdot \frac{\partial u}{\partial x}+\frac{x}{t^{2}}+\frac{12}{t}, \\
u(x, 0)=0, \quad u_{t}(x, 0)=\frac{x}{2}, \quad u_{t t}(x, 0)=2 .
\end{gathered}
$$

Application of inverse operator developed in (15) with $n=$ 2, that is, $L_{t}^{-1}(\cdot)=\int_{0}^{t}\left(1 / t^{2}\right) \iint_{0}^{t} t^{2}(\cdot) d t d t$, on the third-order singular nonlinear PDE of (28) gives

$$
u(x, t)=\frac{x t}{2}+t^{2}+L_{t}^{-1}\left(u_{t t t} \cdot u_{x}\right) .
$$

Now, with the substitution of the dependent variable $u(x, t)$ and the nonlinear term $u_{t t t} \cdot u_{x}$ with the infinite series of (7), (26) becomes

$$
\begin{gathered}
\sum_{m=0}^{\infty} u_{m}(x, t)=\frac{x t}{2}+t^{2}+L_{t}^{-1}\left(\sum_{m=0}^{\infty} A_{m}(x, t)\right) \\
\Longrightarrow\left\{\begin{array}{l}
u_{0}(x, t)=\frac{x t}{2} \\
u_{1}(x, t)=t^{2}+L_{t}^{-1}\left(A_{0}(x, t)\right), \\
u_{m+1}(x, t)=L_{t}^{-1}\left(A_{m}(x, t)\right), \quad m \geq 1 .
\end{array}\right.
\end{gathered}
$$

The Adomian polynomials, $A_{m}$ 's, are obtained as [8]

$$
\begin{gathered}
A_{0}(x, t)=u_{0_{t t t}}(x, t) \cdot u_{0_{x}}(x, t)=0, \\
A_{1}(x, t)=u_{0_{t t t}}(x, t) \cdot u_{1_{x}}(x, t) \\
+u_{1_{t t t}}(x, t) \cdot u_{0_{x}}(x, t)=0, \\
A_{2}(x, t)=u_{0_{t t t}}(x, t) \cdot u_{2_{x}}(x, t)+u_{1_{t t t}}(x, t) \cdot u_{1_{x}}(x, t) \\
+u_{2_{t t t}}(x, t) \cdot u_{0_{x}}(x, t)=0, \\
A_{m}(x, t)=0, \quad m \geq 3 .
\end{gathered}
$$


Also, the expressions for various terms of the solution $u(x, t)$, that is, $u_{m}$ 's, become

$$
\begin{aligned}
& u_{0}(x, t)=\frac{x t}{2}, \\
& u_{1}(x, t)=t^{2}, \\
& u_{2}(x, t)=L_{t}^{-1}\left(A_{1}(x, t)\right)=0, \\
& u_{3}(x, t)=L_{t}^{-1}\left(A_{2}(x, t)\right)=0, \\
& u_{m}(x, t)=0, \quad m \geq 4 .
\end{aligned}
$$

In conclusion, the solution of the third-order singular nonlinear initial-value problem of (28) is

$$
u(x, t)=u_{0}(x, t)+u_{1}(x, t)+\cdots=\frac{x t}{2}+t^{2}
$$

which is the exact solution of (28). It can be seen that the exact solution of (28), (33), is obtained only by the first-order approximation using the new developed inverse differential operator, (15). This reveals that the new developed inverse differential operator is an effective tool in handling the general singular nonlinear IVPs of third-order PDEs.

3.2. Case Study 2. We take into consideration the following singular nonlinear IVP of third-order PDE:

$$
\begin{aligned}
& \frac{\partial^{3} u}{\partial t^{3}}+\frac{9}{t} \frac{\partial^{2} u}{\partial t^{2}}+\frac{18}{t^{2}} \frac{\partial u}{\partial t}+\frac{6}{t^{3}} u=\left(\frac{\partial u}{\partial x}\right)^{2}+x+t, \\
& u(x, 0)=0, \quad u_{t}(x, 0)=0, \quad u_{t t}(x, 0)=0 .
\end{aligned}
$$

The inverse operator $L_{t}^{-1}(\cdot)=\left(1 / t^{3}\right) \iiint_{0}^{t} t^{3}(\cdot) d t d t d t$, defined in (23) with $n=3$, is applied to the PDE of (34), which gives

$$
u(x, t)=\frac{x t^{3}}{120}+\frac{t^{4}}{210}+L_{t}^{-1}\left(\left(u_{x}\right)^{2}\right) .
$$

Due to the MDM, with the use of the infinite series of (7), (35) can be rewritten as

$$
\begin{gathered}
\sum_{m=0}^{\infty} u_{m}(x, t)=\frac{x t^{3}}{120}+\frac{t^{4}}{210}+L_{t}^{-1}\left(\sum_{m=0}^{\infty} A_{m}(x, t)\right) \\
\Longrightarrow\left\{\begin{array}{l}
u_{0}(x, t)=\frac{x t^{3}}{120}, \\
u_{1}(x, t)=\frac{t^{4}}{210}+L_{t}^{-1}\left(A_{0}(x, t)\right), \\
u_{m+1}(x, t)=L_{t}^{-1}\left(A_{m}(x, t)\right), \quad m \geq 1 .
\end{array}\right.
\end{gathered}
$$

By doing computations in the symbolic software Mathematica, the $A_{m}$ 's are obtained as [8]

$$
\begin{gathered}
A_{0}(x, t)=\left(u_{0_{x}}(x, t)\right)^{2}=\frac{t^{3}}{14400}, \\
A_{1}(x, t)=2 u_{0_{x}}(x, t) \cdot u_{1_{x}}(x, t)=0, \\
A_{2}(x, t)=2 u_{0_{x}}(x, t) \cdot u_{2_{x}}(x, t)+\left(u_{1_{x}}(x, t)\right)^{2}=0, \\
A_{m}(x, t)=0, \quad m \geq 3 .
\end{gathered}
$$

The $u_{m}$ 's are also obtained as follows:

$$
\begin{aligned}
& u_{0}(x, t)=\frac{x t^{3}}{120}, \\
& u_{1}(x, t)=\frac{t^{4}}{210}+\frac{t^{9}}{19008000}, \\
& u_{2}(x, t)=L_{t}^{-1}\left(A_{1}(x, t)\right)=0, \\
& u_{3}(x, t)=L_{t}^{-1}\left(A_{2}(x, t)\right)=0, \\
& u_{m}(x, t)=0, \quad m \geq 4 .
\end{aligned}
$$

Therefore, the solution of the singular initial-value problem of (34) is obtained as follows:

$$
u(x, t)=u_{0}(x, t)+u_{1}(x, t)+\cdots=\frac{x t^{3}}{120}+\frac{t^{4}}{210}+\frac{t^{9}}{19008000}
$$

which is the exact solution of (34). Here, the exact solution, (39), is obtained using the new inverse operator developed in Section 2.3, (23). This fact indicates that the newly developed inverse differential operator is very efficient in solving the general complete singular nonlinear IVPs of third-order PDEs.

\section{Conclusions}

The modified decomposition method (MDM) in conjunction with a few proposed inverse differential operators is used to solve the singular nonlinear initial-value problems (IVPs) of third-order PDEs. The new MDM, called the improved MDM (IMDM), is applied on two case studies for third-order singular nonlinear IVPs, for which the IMDM analytical solutions are obtained and seen to be the same as exact analytical solutions. The fast rate of convergence of the IMDM results towards the exact solutions indicates that the IMDM is a very efficient method for handling the third-order singular nonlinear PDEs.

\section{Conflict of Interests}

The author declares that there is no conflict of interests regarding the publication of this paper.

\section{References}

[1] W. F. Ames, Nonlinear Partial Differential Equations in Engineering, Academic Press, New York, NY, USA, 1972.

[2] L. Debnath, Nonlinear Partial Differential Equations for Scientists and Engineers, Birkhäuser, Berlin, Germany, 1997.

[3] A. D. Polyanin and V. F. Zaitsev, Handbook of Nonlinear Partial Differential Equations, Chapman and Hall, New York, NY, USA, 2003.

[4] F. C. Cirstea, Nonlinear methods in the study of singular partial differential equations [Ph.D. thesis], Victoria University of Technology, Victoria, Australia, 2004.

[5] A. M. Wazwaz, Partial Differential Equations and Solitary Wave Theory, Springer, New York, NY, USA, 2009. 
[6] A. M. Wazwaz, "Construction of soliton solutions and periodic solutions of the Boussinesq equation by the modified decomposition method," Chaos, Solitons and Fractals, vol. 12, no. 8, pp. 1549-1556, 2001.

[7] A.-M. Wazwaz, "The modified decomposition method and Padé approximants for a boundary layer equation in unbounded domain," Applied Mathematics and Computation, vol. 177, no. 2, pp. 737-744, 2006.

[8] A.-M. Wazwaz, "The modified decomposition method for analytic treatment of differential equations," Applied Mathematics and Computation, vol. 173, no. 1, pp. 165-176, 2006.

[9] S. A. Kechil and I. Hashim, "Non-perturbative solution of freeconvective boundary-layer equation by Adomian decomposition method," Physics Letters A: General, Atomic and Solid State Physics, vol. 363, no. 1-2, pp. 110-114, 2007.

[10] Y. Q. Hasan and L. M. Zhu, "Modified adomian decomposition method for singular initial value problems in the second order ordinary differential equations," Surveys in Mathematics and its Applications, vol. 3, pp. 183-193, 2008.

[11] A.-M. Wazwaz, "The combined Laplace transform-Adomian decomposition method for handling nonlinear Volterra integro-differential equations," Applied Mathematics and Computation, vol. 216, no. 4, pp. 1304-1309, 2010.

[12] A.-M. Wazwaz and M. S. Mehanna, "The combined LaplaceAdomian method for handling singular integral equation of heat transfer," International Journal of Nonlinear Science, vol. 10, no. 2, pp. 248-252, 2010.

[13] T. R. Sivakumar and S. Baiju, "Shooting type Laplace-Adomian decomposition algorithm for nonlinear differential equations with boundary conditions at infinity," Applied Mathematics Letters, vol. 24, no. 10, pp. 1702-1708, 2011.

[14] A. Noghrehabadi, M. Ghalambaz, and A. Ghanbarzadeh, "A new approach to the electrostatic pull-in instability of nanocantilever actuators using the ADM-Padé technique," Computers \& Mathematics with Applications, vol. 64, no. 9, pp. 2806-2815, 2012.

[15] J.-S. Duan and R. Rach, "Higher-order numeric Wazwaz-ElSayed modified Adomian decomposition algorithms," Computers \& Mathematics with Applications, vol. 63, no. 11, pp. 15571568, 2012.

[16] Y. Lin, Y. Liu, and Z. Li, "Symbolic computation of analytic approximate solutions for nonlinear fractional differential equations," Computer Physics Communications, vol. 184, no. 1, pp. 130-141, 2013.

[17] M. M. Kermani and M. Dehestani, "Solving the nonlinear equations for one-dimensional nano-sized model including Rydberg and Varshni potentials and Casimir force using the decomposition method," Applied Mathematical Modelling, vol. 37, no. 5, pp. 3399-3406, 2013.

[18] L. Song and W. Wang, "A new improved Adomian decomposition method and its application to fractional differential equations," Applied Mathematical Modelling, vol. 37, no. 3, pp. 1590-1598, 2013. 


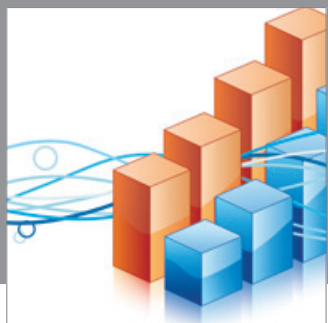

Advances in

Operations Research

mansans

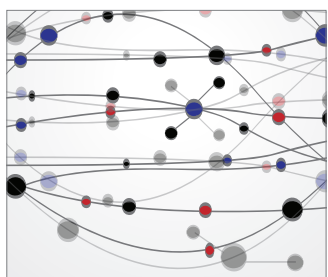

The Scientific World Journal
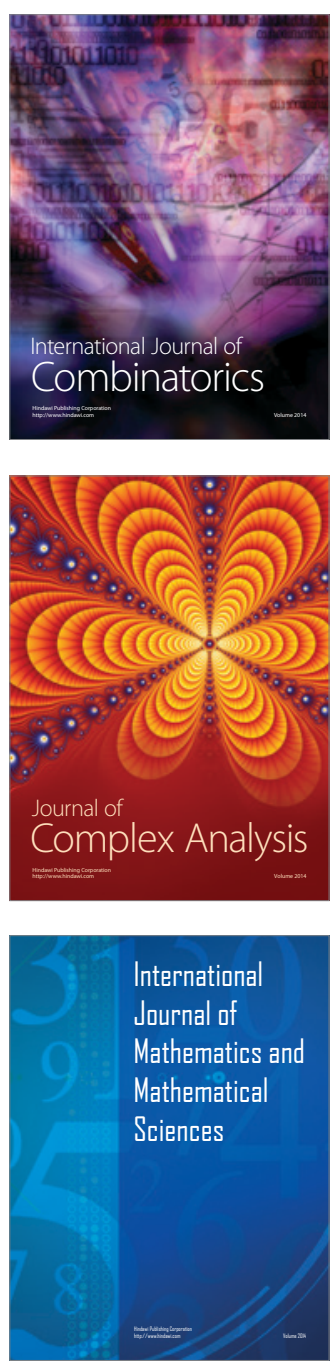
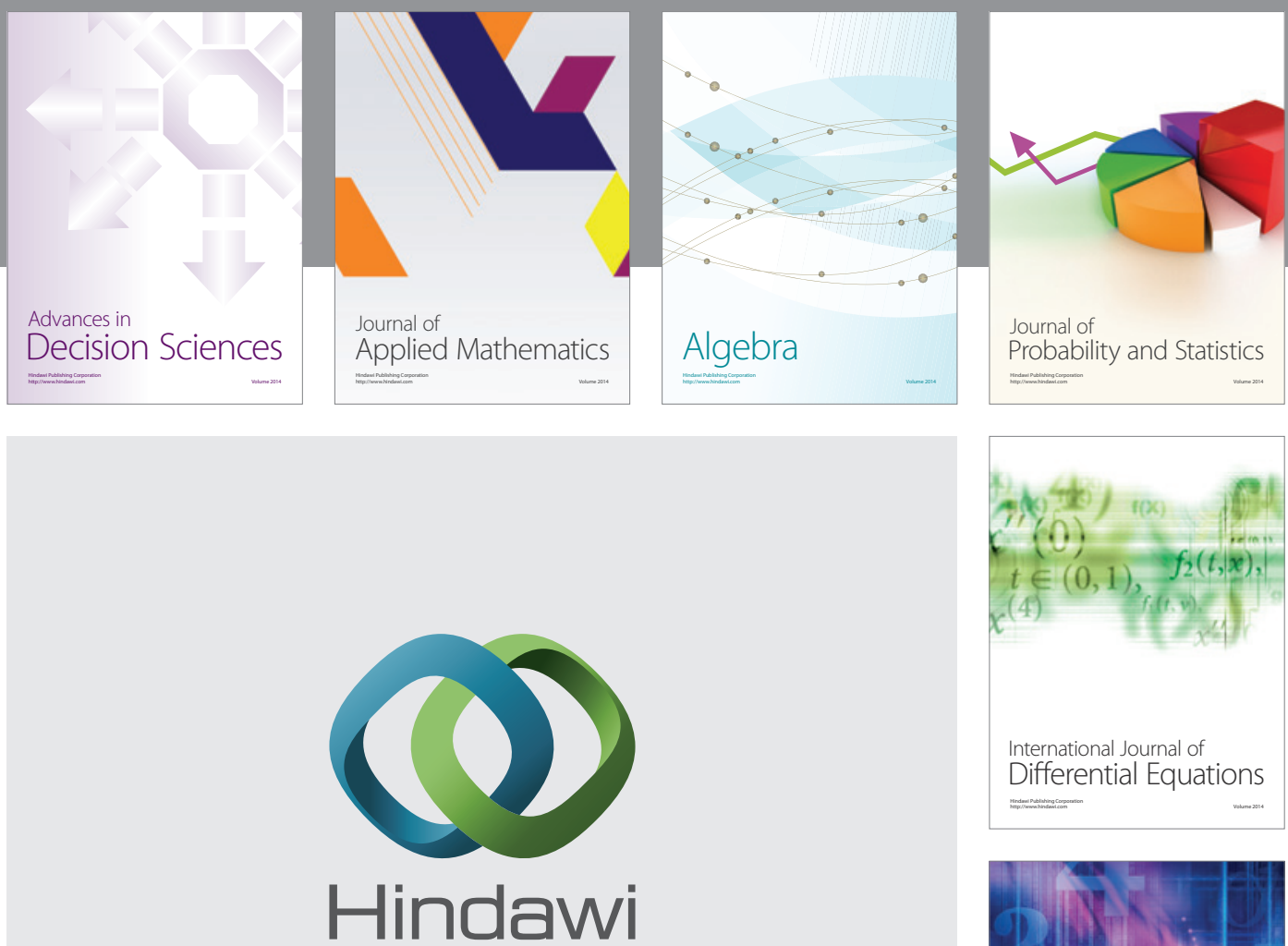

Submit your manuscripts at http://www.hindawi.com
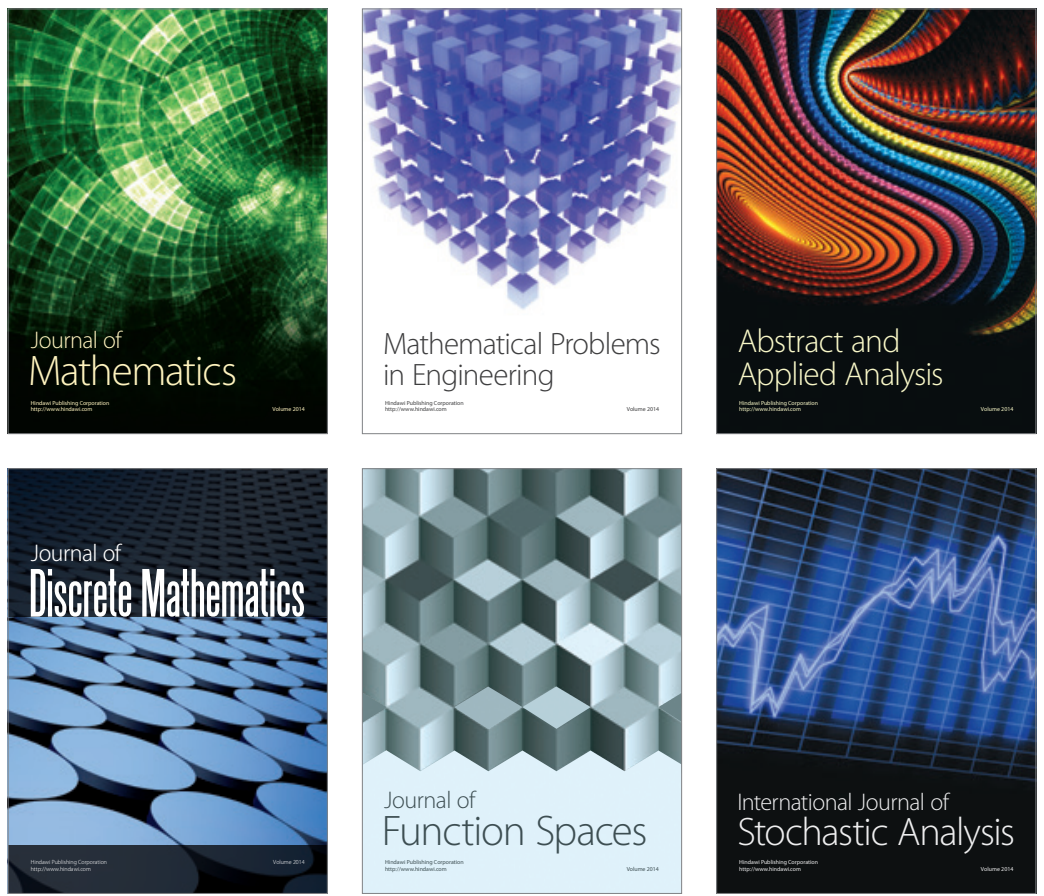

Journal of

Function Spaces

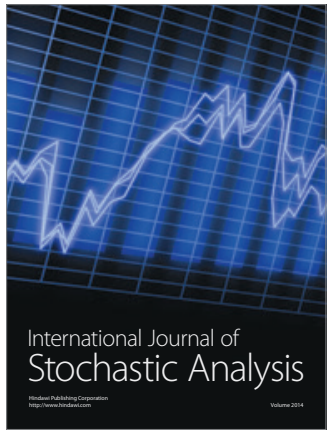

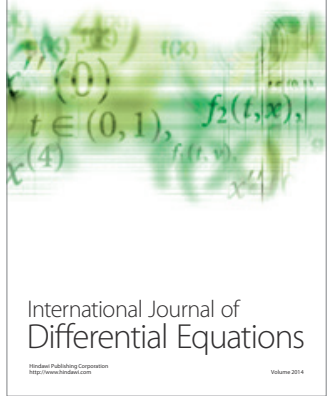
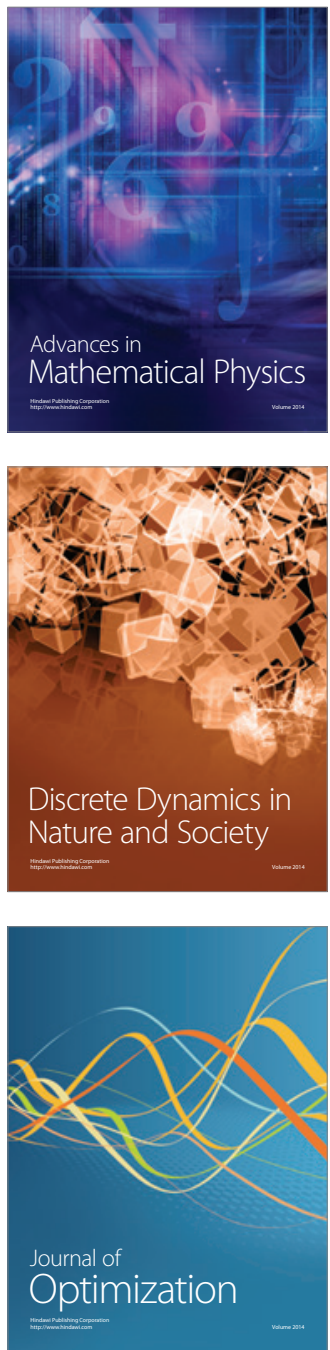